\title{
Candida lusitaniae Breakthrough Fungemia in an Immuno-Compromised Adolescent: Case Report and Review of the Literature
}

\author{
Athanasia Apsemidou 1® Miriam Antonie Füller ${ }^{2}$, Evgeny A. Idelevich ${ }^{3,4}$, Oliver Kurzai ${ }^{5,6}$, \\ Athanasios Tragiannidis ${ }^{1,2}$ (D) and Andreas H. Groll ${ }^{2, *}$ (D) \\ 1 2nd Pediatric Department, Aristotle University of Thessaloniki, AHEPA Hospital, \\ GR-54636 Thessaloniki, Greece; sissyaps@gmail.com (A.A.); atragian@auth.gr (A.T.) \\ 2 Infectious Disease Research Program, Center for Bone Marrow Transplantation and Department of Pediatric \\ Hematology and Oncology, University Children's Hospital Münster, D-48149 Münster, Germany; \\ MiriamAntonie.Fueller@ukmuenster.de \\ 3 Institute of Medical Microbiology, University Hospital Münster, D-48149 Münster, Germany; \\ Evgeny.Idelevich@med.uni-greifswald.de \\ 4 Friedrich Loeffler Institute of Medical Microbiology, University Medicine Greifswald, \\ D-17475 Greifswald, Germany \\ 5 National Reference Center for Invasive Mycoses, Leibniz Institute for Natural Product Research and \\ Infection Biology-Hans-Knoell-Institute, D-07745 Jena, Germany; okurzai@hygiene.uni-wuerzburg.de \\ 6 Institute for Hygiene and Microbiology, Julius Maximilian University of Würzburg, \\ D-97080 Würzburg, Germany \\ * Correspondence: grollan@ukmuenster.de; Tel.: +49-251-834-7742; Fax: +49-251-834-7828
}

Received: 28 November 2020; Accepted: 18 December 2020; Published: 21 December 2020

\begin{abstract}
Candida lusitaniae is a rare cause of candidemia that is known for its unique capability to rapidly acquire resistance to amphotericin B. We report the case of an adolescent with grade IV graft-vs.-host disease after hematopoietic cell transplantation who developed catheter-associated C. Iusitaniae candidemia while on therapeutic doses of liposomal amphotericin B. We review the epidemiology of C. lusitaniae bloodstream infections in adult and pediatric patients, the development of resistance, and its role in breakthrough candidemia. Appropriate species identification, in vitro susceptibility testing, and source control are pivotal to optimal management of $C$. lusitaniae candidemia. Initial antifungal therapy may consist of an echinocandin and be guided by in vitro susceptibility and clinical response.
\end{abstract}

Keywords: Candida lusitaniae; candidemia; resistance; breakthrough; infection; transplantation

\section{Introduction}

Invasive opportunistic fungal diseases caused by Candida spp. are important causes of morbidity and mortality in immunocompromised pediatric patients, including those with cancer and following hematopoietic cell transplantation (HCT). Whereas Candida albicans has long been the most common species isolated from blood cultures, there is a steady increase in fungal infections caused by non-albicans Candida species [1,2]. Among the non-albicans Candida species, Candida lusitaniae is an uncommon pathogen that accounts for approximately $1 \%$ of isolates in large datasets of adult and pediatric patients with candidemia and other forms of invasive candidiasis [1,3]. However, apart from its potential to be intrinsically resistant, this Candida species is of special interest as it is capable of rapidly acquiring resistance to amphotericin B and can cause breakthrough infections or treatment failure in immunocompromised patients [4-7]. This report describes the case of a severely immunocompromised 
17-year-old adolescent allogeneic HCT recipient with late onset grade IV graft-vs.-host disease (GvHD) and catheter-associated C. lusitaniae candidemia occurring under long-term treatment with therapeutic doses of liposomal amphotericin B.

\section{Case Report}

The patient was a 17-year-old male adolescent with precursor $\mathrm{T}$ acute lymphoblastic leukemia (ALL) and persistent molecular disease who had received an allogeneic bone marrow transplant (HSCT) from a matched unrelated donor after standard conditioning with total body irradiation, high dose etoposide, and anti-thymocyte globuline in October 2015. Post-transplant immunosuppression consisted of three doses of methotrexate $10 \mathrm{mg} / \mathrm{msqu}$ on days 1,3 , and 6 , and cyclosporine A with a target trough concentration of 120-140 ng/mL. Following discharge with a newly implanted Port-a-Cath (Smiths Medical, Dublin, OH, USA) central venous device on day +40 post-transplant, the further course in the outpatient setting was essentially unremarkable until the end of March 2016, when the patient developed late-onset grade IV graft versus host disease (GvHD) of the skin and the gastrointestinal tract during cyclosporine taper, necessitating in-patient management.

Since adjustment of cyclosporine dosage and initiation of high-dose $(2 \mathrm{mg} / \mathrm{kg} /$ day $)$ methylprednisolone failed to induce a response, the patient subsequently received three courses of weekly rituximab (4 April-2 May) in combination with three courses of basiliximab and infliximab (13 April-14 May), while baseline immunosuppression with cyclosporine A (target trough concentration: $120-140 \mathrm{ng} / \mathrm{mL}$ ) and methylprednisolone (1 mg/kg/day) was continued. In addition to parenteral nutrition, the patient received trimethoprim/sulfamethoxazole $160 \mathrm{mg}$ twice daily (BID) on two days per week and valaciclovir $500 \mathrm{mg}$ BID for prophylaxis, as well as meropenem $1000 \mathrm{mg}$ three times daily (TID) plus linezolid $600 \mathrm{mg}$ BID and liposomal amphotericin B $3 \mathrm{mg} / \mathrm{kg} /$ day as empirical therapy for intermittent fever of unknown origin.

On the 3rd of May, while the patient was on empirical antibacterial and antifungal therapy and intermittently granulocytopenic (absolute neutrophil count $(\mathrm{ANC})<500$ cells/uL) for more than four weeks, he developed a new fever episode with a maximum temperature of $38.7^{\circ} \mathrm{C}$. The ANC on that day was 690 cells $/ \mathrm{mL}$, the hemoglobin was $7.7 \mathrm{~g} / \mathrm{dL}$, the platelet count was $16.000 / \mathrm{uL}$, and the c-reactive protein (CRP) was $0.5 \mathrm{mg} / \mathrm{dL}$. Total serum bilirubin was $1.7 \mathrm{mg} / \mathrm{dL}$, and aspartate aminotransferase (AST), alanine aminotransferase (ALT), and alkaline phosphatase were 58, 62, and $169 \mathrm{U} / \mathrm{L}$, respectively. Serum creatinine, blood urea nitrogen (BUN), serum albumin, serum electrolytes, and blood sugar were within normal limits. Blood cultures taken during the initial fever episode remained sterile for bacteria but grew yeasts that were subsequently identified as Candida lusitaniae by MALDI-TOF MS and confirmed by sequencing of the ITS1-5.8S-ITS2 region [8]. Susceptibility testing was performed by broth microdilution according to the EUCAST reference method $[9,10]$, revealing increased minimum inhibitory concentration (MIC) of amphotericin B and no evidence for reduced susceptibility towards azoles or echinocandins (Table 1), with the theoretical limitation that resistance testing of sessile C. lusitaniae cells in biofilms was not performed. Treatment with caspofungin at a dose of $50 \mathrm{mg} / \mathrm{day}$ (day 1: $70 \mathrm{mg}$ ) was initiated on 6 May and liposomal amphotericin B continued at $3 \mathrm{mg} / \mathrm{kg} /$ day to maintain coverage for filamentous fungi. 
Table 1. In vitro susceptibility of the patient's isolates.

\begin{tabular}{cccc}
\hline \multirow{2}{*}{ Antifungal Agent } & \multicolumn{2}{c}{ Minimum Inhibitory Concentration (mg/L) } \\
\cline { 2 - 4 } & $\begin{array}{c}\text { Day 1 Isolate } \\
\text { JMRC:NRZ:0688 }\end{array}$ & $\begin{array}{c}\text { Day 5 Isolate } \\
\text { JMRC:NRZ:0689 }\end{array}$ & $\begin{array}{c}\text { Day 5 Isolate } \\
\text { JMRC:NRZ:0690 }\end{array}$ \\
\hline Amphotericin B & 1 & 1 & 2 \\
\hline Fluconazole & $\leq 0.125$ & $\leq 0.125$ & $\leq 0.125$ \\
\hline Itraconazole & 0.125 & 0.125 & 0.125 \\
\hline Voriconazole & $\leq 0.016$ & $\leq 0.016$ & $\leq 0.016$ \\
\hline Posaconazole & $\leq 0.016$ & $\leq 0.016$ & $\leq 0.016$ \\
\hline Anidulafungin & 0.06 & 0.03 & 0.03 \\
\hline Caspofungin & 0.5 & 0.5 & 1 \\
\hline
\end{tabular}

For details on clinical breakpoints, please see references [10-12]. Day 5 isolates stemmed from different blood culture bottles; the MIC of amphotericin B of isolate 0690 was above the epidemiological cut off value (ECV) of $1 \mathrm{mg} / \mathrm{L}$ [11], and the MIC of caspofungin was above the ECV of $0.5 \mathrm{mg} / \mathrm{L}$ [12], indicating reduced susceptibility to both agents.

While the patient remained hemodynamically stable, he had persistent intermitting fever episodes that were monitored by daily blood cultures. Whereas there was no growth on days 2 to 4 , blood cultures obtained on day 5 became positive for $C$. lusitaniae with an in vitro susceptibility pattern similar to the initial isolate (Table 1). Blood counts, blood sugar, serum electrolytes, and parameters of renal and hepatic function were essentially unchanged, and the CRP was $0.9 \mathrm{mg} / \mathrm{dL}$ (maximum: 3.8 on day 3). At this point, on day 7 (9 May), caspofungin was discontinued and antifungal therapy switched to intravenous fluconazole (400 mg once daily (QD); day 1: $800 \mathrm{mg}$ ) plus liposomal amphotericin B $3 \mathrm{mg} / \mathrm{kg} / \mathrm{day}$, with the indwelling central venous catheter (CVC) finally being removed on day 10 (12 May) (Figure 1). Following defervescence and exclusion of intraocular and intraabdominal lesions, antifungal treatment with fluconazole and liposomal amphotericin $B$ was continued for 10 days after the last positive blood culture (17 May) and then replaced by oral posaconazole (300 mg QD; day 1: $300 \mathrm{mg}$ BID of the gastroresistant tablets) as antifungal prophylaxis (Figure 1). Sadly, 1 month after completion of antifungal therapy and while in partial remission of active GvHD, the patient succumbed to a catheter-related Gram-negative bacterial bloodstream infection with sepsis and refractory septic shock without apparent causal relationship to the prior episode of $C$. lusitaniae fungemia and its treatment.

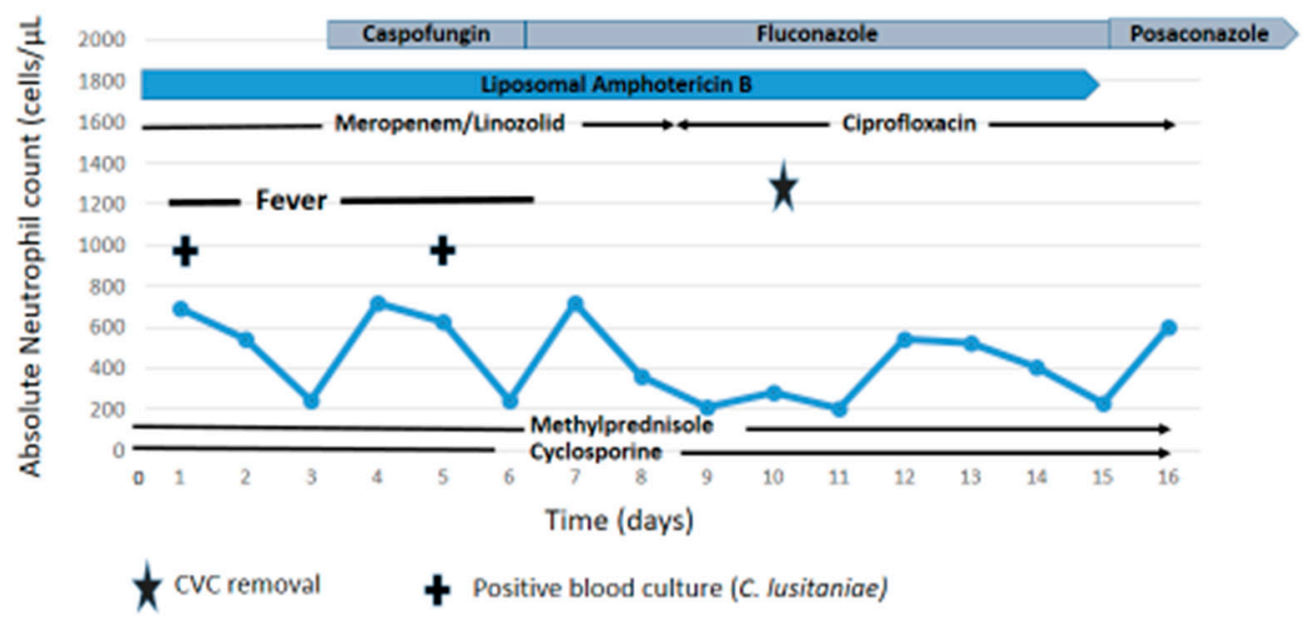

Figure 1. Time course of Candida lusitaniae fungemia and antifungal management. 


\section{Discussion}

While usually appearing susceptible to amphotericin B, the triazoles, and the echinocandins in vitro [1,13-15], C. lusitaniae is different from most medically important Candida species as it can readily develop in vivo resistance to amphotericin B upon exposure to this agent. Apart from mutations in the ergosterol biosynthetic pathway that may have direct effects on gene expression in this haploid yeast, selective gene expression in the adaptive response to amphotericin B may occur with high frequency reversible phenotypic switching from susceptibility to resistance associated with distinct morphologies $[5,16-18]$. Rapid switching to a resistant phenotype with markedly reduced fungicidal activity has been induced in vitro in susceptible strains from patients with failure of amphotericin B-based treatment, which may contribute to the lack of consistent correlations between in vitro susceptibility and responses in vivo [5]. Development of cross resistance to fluconazole in the switched phenotype [19] and of multidrug resistance with accumulation of mutations conferring resistance to all antifungal agents $[6,7]$ have been observed in individual patients. These observations illustrate the potential of C. lusitaniae to rapidly adapt to drug pressure within the host and emphasize the particular need for careful monitoring of antifungal susceptibility and treatment responses.

Dedicated clinical data on invasive C. lusitaniae infections are scarce. In a literature review published in 2003, a total of 55 cases were reported with a predominance of bloodstream infections $(80 \%)$. Although three-fourths of the patients had serious underlying medical conditions, attributable mortality was low (5\%). A total of $5(21.7 \%)$ of 23 isolates with susceptibility testing data were resistant to amphotericin B; two of the affected patients were cured with amphotericin B treatment, and all five survived [20]. In a retrospective analysis conducted at M. D. Anderson Cancer Center in Houston, Texas, between 1988 and 1999, 12 cases of C. lusitaniae candidemia were identified, of which 7 occurred as breakthrough infection ( $58 \%$; four on amphotericin B and three on fluconazole). Eight patients had hematologic malignancies or were post-HCT, and most patients $(75 \%)$ were granulocytopenic at the time of infection. Amphotericin B alone failed in three of six patients; fluconazole alone was effective in three patients with solid tumors, and amphotericin B plus fluconazole was effective in two of three patients. None of the nine isolates available from five patients were resistant in vitro (MIC $>1 \mathrm{mg} / \mathrm{L}$ ) to amphotericin B. The case fatality rate was $25 \%$ [21]. In a subsequent study from the same institution covering 1998 through 2013, C. lusitaniae was isolated in 19 of 1395 Candida bloodstream isolates $(1.4 \%$; or $28 \%$ among 68 patients with fungemia by uncommon Candida species, including Candida guilliermondii, Candida lusitaniae, Candida kefyr, Candida famata, and Candida dubliniensis), with a significant increase over time. Most patients had hematologic malignancies (75\%), 18 were post-HCT (27\%), and 40 (58.8\%) were severely granulocytopenic; a total of 7 of the 19 episodes (36.8\%) occurred as breakthrough infections. All 19 isolates had an MIC of amphotericin B of $\leq 1 \mathrm{mg} / \mathrm{L}$, and 3 of 14 tested isolates had an MIC of caspofungin of $>1 \mathrm{mg} / \mathrm{L}$ as assessed by the Clinical Laboratory Standards Institute broth microdilution reference method [22]. The all-cause 28-day mortality rate was 53\% and not different from the entire cohort [23].

Two large epidemiological studies have been conducted in pediatric patients. In a prospective, multicenter observational study of invasive candidiasis conducted by the International Pediatric Fungal Network between 2007 and 2011, C. lusitaniae accounted for 8 of 201 isolates (4\%) collected from 196 non-neonatal pediatric patients; two of the eight cases were breakthrough infections [24]. In a similar multinational but retrospective study of candidemia conducted by the European Pediatric Mycology Network covering 2005 through 2015, C. lusitaniae was found in less than 2\% of the 1395 cases of candidemia collected in pediatric patients of all age groups [25]. Data obtained from our own institution, the University Children's Hospital Münster, between 1998 and 2016, revealed 56 episodes of candidemia in patients below 20 years. The majority were immunocompromised or critically ill $(75 \%)$, had an indwelling central venous catheter $(92 \%)$, and were receiving broad spectrum antibacterial agents (94\%) at diagnosis. Non-albicans Candida species accounted for $52 \%$ of the isolates, with Candida parapsilosis accounting for $19.6 \%$, Candida glabrata for $10.7 \%$, and C. lusitaniae for $7.1 \%$. Of the four primary isolates of $C$. lusitaniae, two were resistant to amphotericin, two to fluconazole, 
and one was resistant to 5 -fluorocytosine; one of the four cases (the presented case) was a breakthrough infection under amphotericin B therapy $[26,27]$.

Breakthrough fungal infections have been recognized as an emerging problem $[28,29]$ and are defined as any invasive fungal infection occurring during exposure to a systemic antifungal agent, irrespective of its spectrum of activity [30]. In a retrospective study conducted between 2011 and February 2016 in patients $>12$ years admitted to two tertiary care hospitals in Southern Brazil, 27 breakthrough episodes (18\%) were identified among 148 candidemia episodes. Breakthrough infections were associated with neutropenia and mucositis by multivariate analysis and showed a predominance of Candida non-albicans species $(85 \% ; p<0.001)$. There was no clear pattern of resistance in the breakthrough isolates, and no difference was observed in 30 days mortality; C. lusitaniae was not observed among the breakthrough infections [29]. In a retrospective single center study conducted in a pediatric tertiary care center in Taiwan from 2003 through 2015, 45 of 319 episodes of candidemia (14.1\%) were breakthrough infections. Similar to the previous study, the majority of breakthrough candidemia was caused by non-albicans Candida species $(73.3 \% ; p<0.01)$, but only 5 of 43 isolates studied (11.6\%) were resistant to the antifungal agent used at the time of breakthrough. Isolation of $C$. lusitaniae was sporadic $(<4 \%)$, without apparent differences in breakthrough relative to non-breakthrough candidemia. By multivariate analysis, previous azole exposure, neutropenia, and recurrent candidemia were independent risk factors of breakthrough candidemia; episodes of breakthrough candidemia had significantly higher illness severity $(p<0.01)$ and higher rates of attributable mortality $(p<0.001)$ [31].

The patient presented here had classical risk factors for breakthrough candidemia, including hematologic malignancy, status post-allogeneic matched unrelated donor (MUD) HCT, and severe GvHD, prolonged granulocytopenia, impaired mucosal barriers, profound immunosuppression including glucocorticosteroids, presence of a central venous catheter, and long-term use of broad-spectrum antibacterial agents [32]. Similar to the findings in the two larger studies described above $[29,31]$, the isolate belonged to the group of non-albicans Candida species and, in this specific case, was phenotypically resistant to amphotericin $B$, which had been used as empirical antifungal treatment over prolonged periods of time. However, given the clinical predominance of predisposing host and iatrogenic factors, the finding of antifungal resistance and its role in the pathogenesis of this infection are difficult to assess [32]. Clinical non-response to caspofungin, as suggested by persistent fever and two separate positive follow-up blood cultures on day 5 of treatment, most likely was not attributable to failure of the echinocandin but to the lack of source control, as evidenced by prompt improvement and clinical cure following removal of the indwelling central venous catheter. Of note, in an individual patient-level quantitative review of seven randomized trials for treatment of invasive candidiasis including data from 1915 adult patients, removal of a central venous catheter $(p=0.0001)$ and treatment with and echinocandin antifungal $(p=0.02)$ were associated with clinical success and survival [3], leading international guidelines to strongly recommend first-line treatment with an echinocandin and catheter removal for pediatric patients also [33-35].

In summary, the haploid yeast C. Iusitaniae is a rare cause of invasive candidiasis in immunocompromised children and adolescents that may readily develop resistance to amphotericin $\mathrm{B}$ and, potentially, the triazoles and the echinocandins. Careful attention to appropriate species identification, discriminative in vitro susceptibility testing, and strict adherence to the principle of source control are pivotal to optimal management. In the absence of more robust clinical data, initial antifungal therapy should probably consist of an echinocandin and thereafter be guided by the results of in vitro susceptibility testing and clinical response.

Author Contributions: A.A. and M.A.F. prepared the case report; E.A.I. and O.K. were responsible for the microbiological diagnostics; A.A., A.T., and A.H.G. prepared the manuscript draft. All authors have read and agreed to the published version of the manuscript.

Funding: This research received no external funding. 
Acknowledgments: The patient's blood culture isolates have been deposited as isolates JMRC:NRZ:0688, 0689, and 0690 at the Jena Microbial Resource Collection of the Leibniz Institute for Natural Product Research and Infection Biology - Hans Knöll Institute (HKI), Jena, Germany. The work of the National Reference Center for Invasive Mycoses NRZMyk is supported by the Robert Koch Institute from funds provided by the German Ministry of Health (grant 1369-240 to O.K.).

Conflicts of Interest: The authors declare no conflict of interest.

\section{References}

1. Pfaller, M.A.; Andes, D.R.; Diekema, D.J.; Horn, D.L.; Reboli, A.C.; Rotstein, C.; Franks, B.; Azie, N.E. Epidemiology and outcomes of invasive candidiasis due to non-albicans species of Candida in 2496 patients: Data from the Prospective Antifungal Therapy (PATH) registry 2004-2008. PLoS ONE 2014, 9, e101510. [CrossRef]

2. Tragiannidis, A.; Tsoulas, C.; Groll, A.H. Invasive candidiasis and candidaemia in neonates and children: Update on current guidelines. Mycoses 2015, 58, 10-21. [CrossRef] [PubMed]

3. Andes, D.R.; Safdar, N.; Baddley, J.W.; Playford, G.; Reboli, A.C.; Rex, J.H.; Sobel, J.D.; Pappas, P.G.; Kullberg, B.J. Mycoses Study Group. Impact of treatment strategy on outcomes in patients with candidemia and other forms of invasive candidiasis: A patient-level quantitative review of randomized trials. Clin. Infect. Dis. 2012, 54, 1110-1122. [CrossRef] [PubMed]

4. Pfaller, M.A.; Diekema, D.J. Rare and emerging opportunistic fungal pathogens: Concern for resistance beyond Candida albicans and Aspergillus fumigatus. J. Clin. Microbiol. 2004, 42, 4419-4431. [CrossRef] [PubMed]

5. Atkinson, B.J.; Lewis, R.E.; Kontoyiannis, D.P. Candida lusitaniae fungemia in cancer patients: Risk factors for amphotericin B failure and outcome. Med. Mycol. 2008, 46, 541-546. [CrossRef] [PubMed]

6. Asner, S.A.; Giulieri, S.; Diezi, M.; Marchetti, O.; Sanglard, D. Acquired Multidrug Antifungal Resistance in Candida lusitaniae during Therapy. Antimicrob. Agents Chemother. 2015, 59, 7715-7722. [CrossRef]

7. Kannan, A.; Asner, S.A.; Trachsel, E.; Kelly, S.; Parker, J.; Sanglard, D. Comparative Genomics for the Elucidation of Multidrug Resistance in Candida lusitaniae. mBio 2019, 10, e02512-e02519. [CrossRef]

8. White, T.J.; Bruns, T.; Lee, S.; Taylor, J. Amplification and Direct Sequencing of Fungal Ribosomal RNA Genes for Phylogenetics. In PCR Protocols-A Guide to methods and Applications; Innis, M.A., Gelfand, D.A., Sminsky, J.J., White, T.J., Eds.; Academic Press: San Diego, CA, USA, 1990; pp. 315-322. Available online: https:/www.sciencedirect.com/science/article/pii/B9780123721808500421?via\%3Dihub (accessed on 20 December 2020).

9. Arendrup, M.C.; Meletiadis, J.; Mouton, J.W.; Lagrou, K.; Hamal, P.; Guinea, J. The Subcommittee on Antifungal Susceptibility Testing (AFST) of the ESCMID European Committee for Antimicrobial Susceptibility Testing (EUCAST) EUCAST DEFINITIVE DOCUMENT E.DEF 7.3.2 April 2020 Method for the Determination of Broth Dilution Minimum Inhibitory Concentrations of Antifungal Agents for Yeasts. Available online: https://www.eucast.org/fileadmin/src/media/PDFs/EUCAST_files/AFST/Files/EUCAST_E_ Def_7.3.2_Yeast_testing_definitive_revised_2020.pdf (accessed on 20 December 2020).

10. The European Committee on Antimicrobial Susceptibility Testing (EUCAST). Overview of Antifungal ECOFFs and Clinical Breakpoints for Yeasts, Moulds and Dermatophytes Using the EUCAST E.Def 7.3, E.Def 9.3 and E.Def 11.0 Procedures. Version 2. 2020. Available online: http://www.eucast.org (accessed on 20 December 2020).

11. Borman, A.M.; Muller, J.; Walsh-Quantick, J.; Szekely, A.; Patterson, Z.; Palmer, M.D.; Fraser, M.; Johnson, E.M. MIC distributions for amphotericin B, fluconazole, itraconazole, voriconazole, flucytosine and anidulafungin and 35 uncommon pathogenic yeast species from the UK determined using the CLSI broth microdilution method. J. Antimicrob. Chemother. 2020, 75, 1194-1205. [CrossRef]

12. Pfaller, M.A.; Diekema, D.J.; Andes, D.; Arendrup, M.C.; Brown, S.D.; Lockhart, S.R.; Motyl, M.; Perlin, D.S.; CLSI Subcommittee for Antifungal Testing. Clinical breakpoints for the echinocandins and Candida revisited: Integration of molecular, clinical, and microbiological data to arrive at species-specific interpretive criteria. Drug Resist. Updat. 2011, 14, 164-176. [CrossRef]

13. Ernst, E.J.; Yodoi, K.; Roling, E.E.; Klepser, M.E. Rates and extents of antifungal activities of amphotericin B, flucytosine, fluconazole, and voriconazole against Candida lusitaniae determined by microdilution, Etest, and time-kill methods. Antimicrob. Agents Chemother. 2002, 46, 578-581. [CrossRef] 
14. Favel, A.; Michel-Nguyen, A.; Datry, A.; Challier, S.; Leclerc, F.; Chastin, C.; Fallague, K.; Regli, P. Susceptibility of clinical isolates of Candida lusitaniae to five systemic antifungal agents. J. Antimicrob. Chemother. 2004, 53, 526-529. [CrossRef] [PubMed]

15. Lockhart, S.R.; Pham, C.D.; Kuykendall, R.J.; Bolden, C.B.; Cleveland, A.A. Candida lusitaniae MICs to the echinocandins are elevated but FKS-mediated resistance is rare. Diagn. Microbiol. Infect. Dis. 2016, 84, 52-54. [CrossRef]

16. Yoon, S.A.; Vazquez, J.A.; Steffan, P.E.; Sobel, J.D.; Akins, R.A. High-frequency, in vitro reversible switching of Candida lusitaniae clinical isolates from amphotericin B susceptibility to resistance. Antimicrob. Agents Chemother. 1999, 43, 836-845. [CrossRef] [PubMed]

17. Peyron, F.; Favel, A.; Michel-Nguyen, A.; Gilly, M.; Regli, P.; Bolmström, A. Improved detection of amphotericin B-resistant isolates of Candida lusitaniae by Etest. J. Clin. Microbiol. 2001, 39, 339-342. [CrossRef] [PubMed]

18. Miller, N.S.; Dick, J.D.; Merz, W.G. Phenotypic switching in Candida lusitaniae on copper sulfate indicator agar: Association with amphotericin B resistance and filamentation. J. Clin. Microbiol. 2006, 44, 1536-1539. [CrossRef] [PubMed]

19. Favel, A.; Michel-Nguyen, A.; Peyron, F.; Martin, C.; Thomachot, L.; Datry, A.; Bouchara, J.P.; Challier, S.; Noël, T.; Chastin, C.; et al. Colony morphology switching of Candida lusitaniae and acquisition of multidrug resistance during treatment of a renal infection in a newborn: Case report and review of the literature. Diagn. Microbiol. Infect. Dis. 2003, 47, 331-339. [CrossRef]

20. Hawkins, J.L.; Baddour, L.M. Candida lusitaniae infections in the era of fluconazole availability. Clin. Infect. Dis. 2003, 36, 14-18. [CrossRef]

21. Minari, A.; Hachem, R.; Raad, I. Candida lusitaniae: A cause of breakthrough fungemia in cancer patients. Clin. Infect. Dis. 2001, 32, 186-190. [CrossRef]

22. CLSI Reference Method for Broth Dilution Antifungal Susceptibility Testing of Yeasts, 4th ed.; M27-S4; Clinical and Laboratory Standards Institute (CLSI): Wayne, PA, USA, 2012.

23. Jung, D.S.; Farmakiotis, D.; Jiang, Y.; Tarrand, J.J.; Kontoyiannis, D.P. Uncommon Candida Species Fungemia among Cancer Patients, Houston, Texas, USA. Emerg. Infect. Dis. 2015, 21, 1942-1950. [CrossRef]

24. Steinbach, W.J.; Roilides, E.; Berman, D.; Hoffman, J.A.; Groll, A.H.; Bin-Hussain, I.; Palazzi, D.L.; Castagnola, E.; Halasa, N.; Velegraki, A.; et al. International Pediatric Fungal Network. Results from a prospective, international, epidemiologic study of invasive candidiasis in children and neonates. Pediatr. Infect. Dis. J. 2012, 31, 1252-1257. [CrossRef]

25. Warris, A.; Pana, Z.D.; Oletto, A.; Lundin, R.; Castagnola, E.; Lehrnbecher, T.; Groll, A.H.; Roilides, E.; Andersen, C.T.; Arendrup, M.C.; et al. EUROCANDY Study Group ${ }^{* *}$; ${ }^{* *}$ EUROCANDY study group:. Etiology and Outcome of Candidemia in Neonates and Children in Europe: An 11-year Multinational Retrospective Study. Pediatr. Infect. Dis. J. 2020, 39, 114-120. [CrossRef] [PubMed]

26. Tragiannidis, A.; Fegeler, W.; Rellensmann, G.; Debus, V.; Müller, V.; Hoernig-Franz, I.; Siam, K.; Pana, Z.D.; Jürgens, H.; Groll, A.H. Candidaemia in a European Paediatric University Hospital: A 10-year observational study. Clin. Microbiol. Infect. 2012, 18, E27-E30. [CrossRef] [PubMed]

27. Makarova, O.; Tragiannidis, A.; Pana, D.; Idelevich, J.; Becker, K.; Groll, A.H. Candidemia in pediatric patients in a German university hospital: Update from a single center observational cohort study. Mycoses 2016, 59, 10.

28. Lionakis, M.S.; Lewis, R.E.; Kontoyiannis, D.P. Breakthrough Invasive Mold Infections in the Hematology Patient: Current Concepts and Future Directions. Clin. Infect. Dis. 2018, 67, 1621-1630. [CrossRef] [PubMed]

29. Breda, G.L.; Tuon, F.F.; Meis, J.F.; Herkert, P.F.; Hagen, F.; de Oliveira, L.Z.; Dias, V.C.; da Cunha, C.A.; Queiroz-Telles, F. Breakthrough candidemia after the introduction of broad spectrum antifungal agents: A 5-year retrospective study. Med. Mycol. 2018, 56, 406-415. [CrossRef] [PubMed]

30. Cornely, O.A.; Hoenigl, M.; Lass-Flörl, C.; Chen, S.C.; Kontoyiannis, D.P.; Morrissey, C.O.; Thompson, G.R., 3rd; Mycoses Study Group Education and Research Consortium (MSG-ERC); The European Confederation of Medical Mycology (ECMM). Defining breakthrough invasive fungal infection-Position paper of the mycoses study group education and research consortium and the European Confederation of Medical Mycology. Mycoses 2019, 62, 716-729. [CrossRef] [PubMed] 
31. Lai, M.Y.; Hsu, J.F.; Chu, S.M.; Wu, I.H.; Huang, H.R.; Lin, C.C.; Lee, I.T.; Chiang, M.C.; Fu, R.H.; Tsai, M.H. Breakthrough candidemia in children: Clinical and microbiological characteristics, therapeutic strategies and impact on outcomes. Future Microbiol. 2017, 12, 695-705. [CrossRef]

32. Jenks, J.D.; Cornely, O.A.; Chen, S.C.; Thompson, G.R., 3rd; Hoenigl, M. Breakthrough invasive fungal infections: Who is at risk? Mycoses 2020, 63, 1021-1032. [CrossRef]

33. Hope, W.W.; Castagnola, E.; Groll, A.H.; Roilides, E.; Akova, M.; Arendrup, M.C.; Arikan-Akdagli, S.; Bassetti, M.; Bille, J.; Cornely, O.A.; et al. ESCMID Fungal Infection Study Group. ESCMID* guideline for the diagnosis and management of Candida diseases 2012: Prevention and management of invasive infections in neonates and children caused by Candida spp. Clin. Microbiol. Infect. 2012, 18 (Suppl. S7), 38-52. [CrossRef]

34. Groll, A.H.; Castagnola, E.; Cesaro, S.; Dalle, J.H.; Engelhard, D.; Hope, W.; Roilides, E.; Styczynski, J.; Warris, A.; Lehrnbecher, T. Fourth European Conference on Infections in Leukaemia; Infectious Diseases Working Party of the European Group for Blood Marrow Transplantation (EBMT-IDWP); Infectious Diseases Group of the European Organisation for Research and Treatment of Cancer (EORTC-IDG); International Immunocompromised Host Society (ICHS); European Leukaemia Net (ELN). Fourth European Conference on Infections in Leukaemia (ECIL-4): Guidelines for diagnosis, prevention, and treatment of invasive fungal diseases in paediatric patients with cancer or allogeneic haemopoietic stem-cell transplantation. Lancet Oncol. 2014, 15, e327-e340.

35. Pappas, P.G.; Kauffman, C.A.; Andes, D.R.; Clancy, C.J.; Marr, K.A.; Ostrosky-Zeichner, L.; Reboli, A.C.; Schuster, M.G.; Vazquez, J.A.; Walsh, T.J.; et al. Executive Summary: Clinical Practice Guideline for the Management of Candidiasis: 2016 Update by the Infectious Diseases Society of America. Clin. Infect. Dis. 2016, 62, 409-417. [CrossRef] [PubMed]

Publisher's Note: MDPI stays neutral with regard to jurisdictional claims in published maps and institutional affiliations. 